\title{
Mixing Performance of Counter-Axial Flow Impeller using Computational Fluid Dynamics
}

\author{
Ian Torotwa and Changying Ji * \\ College of Engineering, Nanjing Agricultural University Jiangsu Province, Nanjing 210031. P.R. China \\ Received 12 Jan 2018, Accepted 21 March 2018, Available online 22 March 2018, Vol.8, No.2 (March/April 2018)
}

\begin{abstract}
In this study, turbulent flow fields in a baffled vessel stirred by counter-axial flow impeller have been investigated in comparison to the Rushton turbine. The resultant turbulence was numerically predicted using computational fluid dynamics (CFD). Turbulence models were developed in ANSYS Fluent 18.1 solver using the Navier-Stokes equation with the standard $k-\varepsilon$ turbulence model. The Multiple Reference Frame (MRF) approach was used to simulate the impeller action in the vertical and horizontal planes of the stirred fluid volume. Velocity profiles generated from the simulations were used to predict and compare the performance of the two designs. To validate the CFD model, the simulation results were compared with experimental results from existing work and a satisfactory agreement was established. It was concluded that the counter-axial flow impeller could provide better turbulence characteristics that would improve the quality of mixing systems.
\end{abstract}

Keywords: Counter-axial flow impeller, Rushton turbine, Turbulent mixing, Computational Fluid Dynamics, Stirred vessel.

\section{Introduction}

Agitators are used for various industrial operations such as mixing of miscible liquids, dispersion of gases, solids suspension and chemical reactions. Flow behavior in agitation vessels are known to be chaotic and difficult to predict, therefore, attaining uniformity in such mixing processes is a demanding task (Delvigne, et al, 2005).

The study of the flow produced by agitators is very important, as it allows its performance to be determined and optimized in order to realize the desired outcome. This would facilitate the choice of an appropriate design that generates sufficient mixing in the vessel. In order to study the mixing action in stirred vessels, experiments have been undertaken. Such an approach is usually costly and complicated. Using computational fluid dynamics (CFD), various factors affecting the agitation process can be investigated theoretically with less time and costs (Versteeg and Malalasekera, 2007). Improvements can then be made on the design features for best performance. (Sommerfeld and Decker, 2003), observed that CFD has advanced to a level of realizing reliable results when predicting the flow characteristics in stirred vessels.

Since impeller design is one of the most important components of determining the performance of

*Corresponding author's ORCID ID: 0000-0003-4851-7386, DOI: https://doi.org/10.14741/ijcet/v.8.2.14 mechanically agitated mixers (Chun-Yan, et al, 2014), a lot of effort has been devoted to study its mixing efficiency using both experimental and simulation methods. (Kuncewicz and Pietrzykowski, 2006), studied the effect of the impeller diameter on the power number. (Kumaresan and Joshi, 2006), examined the effect of the blade numbers and the axial location of the impeller in the fluid flow in the container. (Vakili and Esfaharry, 2009), considered the effect of geometrical features in the agitated vessels with two-blade impeller. (Aubin and Xuereb, 2006), investigated the use of multiple Intermig impeller design for stirring viscous Newtonian fluids. (Jahoda, et $a l$, 2007), did studies on different simulation approaches for establishing homogenization in mixing tanks.

Among the commonly available impellers, the Rushton turbine has been used for many comparative studies (Musgrove, et al, 2000). In one of such study, (Nienow, 1996), compared its performance to other impellers. (Bonvillani, et al, 2006) experimentally determined the mixing times for a tank equipped with a stirrer propelled by two Rushton turbines.

An in-depth review of literature revealed that no work has been done pertaining to the counter-axial flow impeller. This paper thus focuses on exploring the hydrodynamic behavior of an impeller that performs counter-axial flow mixing. The objective of this work is to use CFD to determine the performance of this nonconventional type of impeller in comparison to the 
Rushton six blade turbine. Simulations were done using ANSYS Fluent 18.1 solver. The standard k-epsilon ( $\varepsilon$ ) model was used to set up the turbulent flow process and the multiple reference frames (MRF) approach was used to model the impeller motion in a baffled tank. Velocity profiles generated in CFD were interpreted as the impeller flow behavior.

\section{Tank Geometry}

A cylindrical tank with four baffles arranged symmetrically on the tank's inner walls was used for the mixing model. The shaft holding the impellers had a diameter of $0.012 \mathrm{~m}$ and positioned concentric to the axis of the tank. Baffles were included in the set-up to prevent liquid vortex. The outline of the experimental tank and the dimensions of the agitation components are shown in Figure 1 and Table 1 respectively. The mixing arrangement was defined in the cylindrical coordinates $(r, \theta, z)$.
Table 1 Dimensions of the agitation apparatus

\begin{tabular}{ccc}
\hline Parameter & Symbol & Value (mm) \\
\hline Tank diameter & $\mathrm{T}$ & 360 \\
Tank height & $\mathrm{H}$ & 500 \\
Impeller diameter & $\mathrm{D}=\mathrm{T} / 2$ & 180 \\
Impeller blade height & $\mathrm{h}$ & 10 \\
Baffle length & $\mathrm{L}$ & 440 \\
Baffle width & $\mathrm{B}=\mathrm{D} / 12$ & 15 \\
Impeller clearance & $\mathrm{C}=\mathrm{D} / 3$ & 60 \\
\hline
\end{tabular}

\subsection{Design Details of the Impellers}

The designs of impellers used for the analysis were prepared in Solid-works 2016 software. The blade thickness of the impellers was $2.5 \mathrm{~mm}$ with a diameter of $0.180 \mathrm{~m}(\mathrm{~T} / \mathrm{D}=0.5)$. The impellers were placed 60 $\mathrm{mm}=\mathrm{D} / 3$ above the tangential line of the tank bottom surface. Figure 2 below shows the design details of the two impellers.

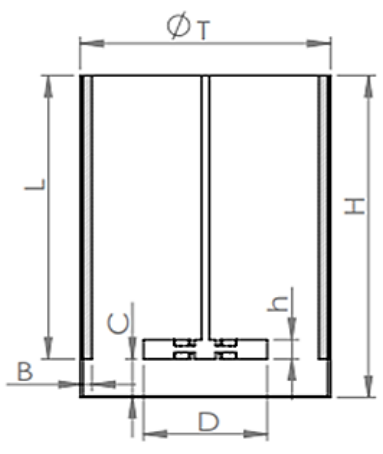

Fig. 1 Mixing tank dimensions

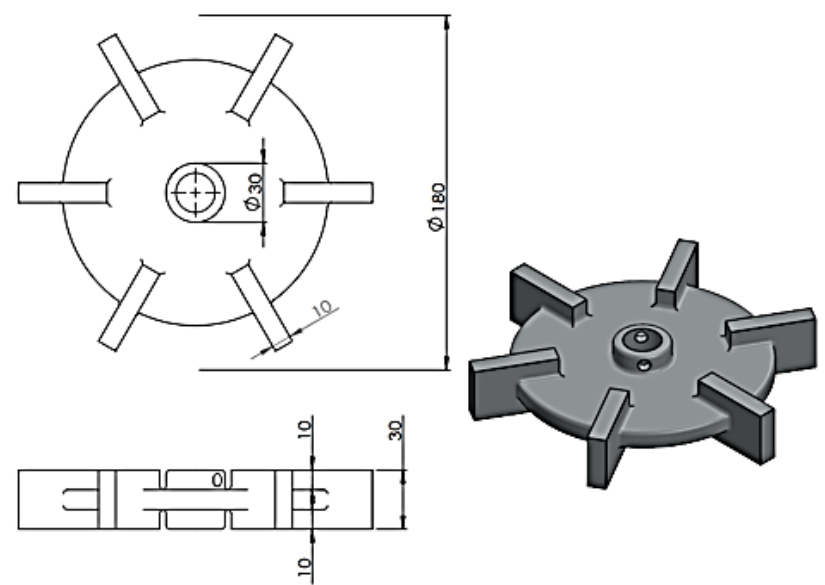

(a)
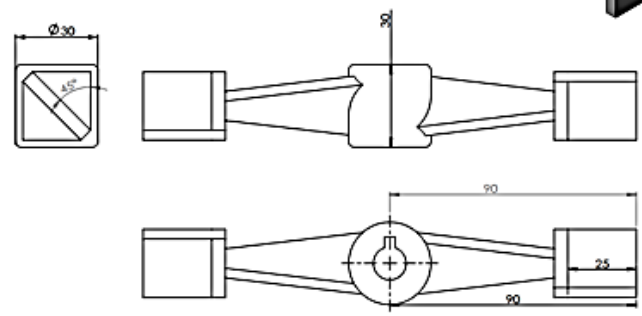

(b)

Fig. 2 (a) Rushton 6-blade impeller and (b) Counter-axial flow impeller designed for the study

The counter-flow impeller design was based on the idea that the blades can be modeled to produce axial flow in opposite directions. This is with the view that the counter flows generated will increase turbulence and mixing performance. The blades consisted of two sections inclined at $45^{\circ}$ to the horizontal and facing opposite directions to each other.

\section{Numerical Modeling}

The aim of turbulence simulation is to predict the physical behavior of turbulent flow generated in a system using numerical methods. Turbulent motions in engineering applications are three-dimensional, nonhomogenous and non-isotropic. The various methods 
of simulating this behavior allows for the statistical description of variable flow fields using the postprocess. A modeling method used should ensure accuracy, simplicity and computational efficiency (Leschziner and Drikakis, 2002).

A number of approaches have been employed for turbulent flow simulation in stirred tanks. In the case of CFD, the Reynolds-averaged Navier-Stokes (RANS) equations, the Large Eddy Simulation (LES) and the Direct Numerical Simulation (DNS) are the three main methods commonly used. In the RANS technique, the equations are averaged over a time interval or across a collection of equivalent fields. RANS computations are extensively used in practical computations for predicting steady-state solutions. Anisotropy in the nature of flow introduces a key uncertainty in the computation.

The Navier-Stokes equations are used to represent the characteristics of turbulence and form the basis of describing the flow phenomena. The chaotic nature of turbulent fluxes act as a direct result of non-linear terms in the $\mathrm{N}-\mathrm{S}$ equations. These equations are based on the conservation laws namely the continuity, momentum and energy conservation laws as respectively given below (ANSYS Fluent User Guide, 2017).

$\frac{\partial \rho}{\partial t}+\nabla \cdot(\rho u)=o$

$\frac{\partial \rho u}{\partial t}+\nabla \cdot(\rho u u)=-\nabla \cdot P$

$\frac{\partial \rho e}{\partial t}+\nabla \cdot(e u)=-\nabla(u \cdot P)-\nabla \cdot q$

Where $u, \rho, e$ and $q$ are the velocity components, density, total energy per unit volume, and heat flux, respectively.

The stress tensor, $P$ for a Newtonian fluid is defined by,

$P=p(\rho, T) I+\frac{2}{3} \mu(\nabla \cdot u) I-\mu\left[(\nabla u)+(\nabla v)^{T}\right]$

Where, $p(\rho, T)$ is the scalar pressure, $I$, is a unit diagonal tensor, $T$ is the temperature, and $\mu$ is the dynamic viscosity coefficient.

Thus, the Navier-Stokes equation (Paul, et al, 2004) can be given by,

$\frac{\partial U_{i}}{\partial t}+U_{j} \frac{\partial U_{i}}{\partial x_{j}}=-\frac{\partial}{\partial x_{i}}\left(\frac{P}{\rho}\right)+\frac{\partial}{\partial x_{j}}\left(v \frac{\partial U_{i}}{\partial x_{j}}\right)$

The k- $\varepsilon$ model is a two-equation method under the RANS approach, where, the turbulent kinetic energy (TKE) and its dissipation rate $(\varepsilon)$ are used to describe the unsteady fields. These two parameters are obtained in the flow field by solving their modeled partial differential transport equations.

The standard $k-\varepsilon$ model solves for high Reynolds number scenarios. This model is formulated on the assumption that the Reynolds stress is proportional to the mean velocity gradient (Dagadu, et al, 2014).

The constant of proportionality is taken to be the eddy viscosity, given as,

$v_{t}=C_{\mu} \cdot \frac{k^{2}}{\varepsilon}$

Where $k$ is the kinetic energy, $\varepsilon$ is the dissipation rate and $C_{\mu}$ is a parameter that depends on the $\mathrm{k}-\varepsilon$ turbulence model.

The equation showing the TKE for three-dimensional flows can be represented as,

$k=\frac{1}{2} \cdot\left(u^{2}+v^{2}+w^{2}\right)$

The governing transport equations ( $k$-equation and $\varepsilon$ equation) for the Standard k- $\varepsilon$ model are given below by equations eight and nine respectively,

$\frac{\partial(\rho k)}{\partial t}+\frac{\partial\left(\rho u_{i} k\right)}{\partial x_{i}}=\frac{\partial\left(\rho u_{i} k\right)}{\partial x_{i}}\left(\frac{\mu_{t}}{\sigma_{k}} \cdot \frac{\partial k}{\partial x_{i}}\right)+\rho \cdot(P-\varepsilon)$

$\frac{\partial(\rho \varepsilon)}{\partial t}+\frac{\partial\left(\rho u_{i} \varepsilon\right)}{\partial x_{i}}=\frac{\partial\left(\rho u_{i} k\right)}{\partial x_{i}}\left(\frac{\mu_{t}}{\sigma_{\varepsilon}} \cdot \frac{\partial \varepsilon}{\partial x_{i}}\right)+\rho \cdot \frac{1}{\tau_{d}} \cdot\left(C_{1, \varepsilon} P-C_{2, \varepsilon} \varepsilon\right)$

Where, $\tau_{d}$ is the dissipation rate time scale that characterizes the dynamic process in the energy spectrum and $P$ is the evolution of turbulence, represented respectively as,

$\tau_{d}=\frac{k}{\varepsilon}$

$P=v_{t}\left(\frac{\partial u_{i}}{\partial x_{j}}+\frac{\partial u_{j}}{\partial x_{i}}\right) \frac{\partial u_{j}}{\partial x_{i}}$

The values of empirical constants of the Standard k- $\varepsilon$ model are $C_{\mu}=0.09, \sigma_{k}=1, \sigma_{\varepsilon}=1.314, C_{1, \varepsilon}=1.44$, and $C_{2, \varepsilon}=1.92$.

The standard $k-\varepsilon$ model combines reasonable accuracy, time economy and robustness for a wide range of turbulent flows. To improve the predictive accuracy of $\mathrm{k}-\varepsilon$ models, more transport equations have been derived. These include the realizable $\mathrm{k}-\varepsilon$ and the $\mathrm{k}-\varepsilon$ RNG (renormalization group) methods.

The realizable $\mathrm{k}-\varepsilon$ model contains an additional state of eddy viscosity and a transport equation for the dissipation derived from an exact equation for the transport of the mean square vorticity variations. A disadvantage of this model is that it produces nonphysical turbulent viscosities in the turbulent viscosity equation. Thus, the use of this model is limited. The $\mathrm{k}-\varepsilon$ RNG model is a method where the smallest eddies are first resolved in the inertial range and then represented in terms of the next smallest eddies. This process continues until a modified set of the Navier Stokes equations is obtained which can then be solved. This approach still poses modeling problems of 
imperfectly solved eddies. Generally, the main weakness associated with the RANS models is that it fails to predict satisfactorily the explicit characteristics of complex flows, since the $k-\varepsilon$ model assumes the isotropy of turbulence. Anisotropic models such as Reynolds Stress Model (RSM), DNS and the LES model have been applied in simulation of complex threedimensional flows.

RSM presents good accuracy in predicting flows with swirl, rotation and high strain rates. It consists of six transport equations for the Reynolds stresses and an equation for the dissipation rate, making it computationally cumbersome. This model also lacks universality in its parameters and it does not adequately capture the time dependent nature of flow. The DNS is based on a three-dimensional and unsteady solution of the Navier-Stokes equations. However, the drawback of this model is its Reynolds number limitations, since the resolution of all the fine scales of a high Reynolds number flow requires enormous computing capability. In addition, it is hard to prove if it yields fully resolved eddies, because it would be impractical with inadequate computing ability.

LES has arisen as a possible choice for modeling, where the time-dependent behavior of the flow is resolved. It is based on the idea that the big eddies produced in the mean flow are anisotropic and have a lengthy lifespan. On the other hand, the small eddies produced from inertial transfer have more universal properties and are isotropic with a short life span hence relatively easy to model. Equations describing this model are derived by filtering the Navier-Stokes equation (Oshinowo and Bakker, 2002). This effectively separates the eddies whose scales are smaller than the filter size used in meshing. The resulting equations have the structure of the original equation and resultant subgrid scale stresses (SGS). The large eddies are resolved directly, while the small eddies are modelled using available subgrid-scale models. The LES model can solve all eddying scales in a complex flow, however, the challenge of limited computing power still prevails, and thus not suitable for practical industrial applications. Moreover, there is excessive dissipation in flows produced by growth of initially small agitation to fully turbulent flow which ought to be resolved. For these reasons, it is deemed that the RANS equations for turbulence modeling are the most fitting CFD tool to use for realistic and economical study of turbulent mixing schemes.

\subsection{CFD Meshing and Pre-Processing}

The impeller configurations, fluid volume and the baffles were modelled as separate regions in solid works 2016 software, before being imported into ANSYS Fluent for pre-processing and meshing. Elaborate interfaces between the contacting fluid regions and boundary conditions were created in ANSYS workbench design modeler.
A mesh was generated to discretize the domains into small control volumes, where the conservation equations were to be approximated by computer numerical calculations. The mesh for the mixing simulation set-up contained two main zones, tank-fluid region and impeller region; modelled as separate interacting fluid domains. An increased mesh density was used near the impeller and the tank walls in order to outline the boundary layer flow details. A fine mesh was used to enhance the stability and accuracy of the computation. Figure 3 below shows the boundary interfaces created and meshed regions of the agitation assembly. A compact mesh can be seen at the impeller and shaft region.
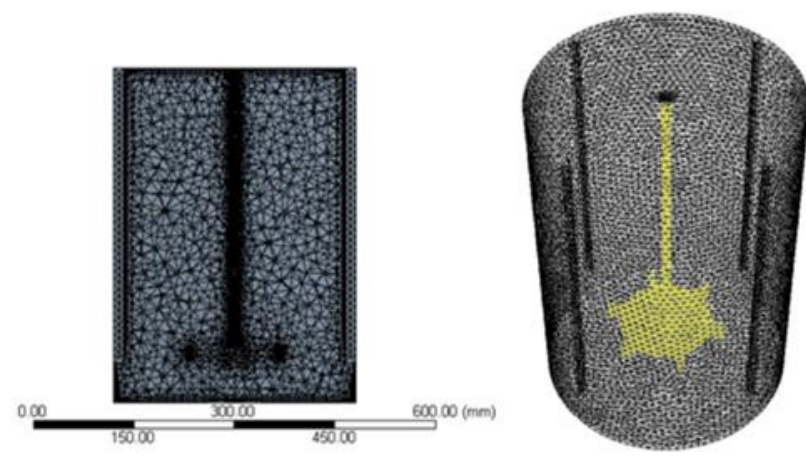

Fig. 3 Boundary conditions of the meshed model

\subsection{Simulation Process and Computation.}

The simulations were prepared in fluent solver, using the pressure-based steady state and absolute velocity conditions with gravity acting in the negative $y$-axis direction. The created fluid regions were set to viscous type in the $\mathrm{k}-\varepsilon$ standard model with standard wall functions. The material was chosen as water-liquid with a density, $\rho$, of $998.2 \mathrm{~kg} / \mathrm{m}^{3}$ and constant viscosity, $\mu$, of $0.001 \mathrm{~kg} / \mathrm{m} . \mathrm{s}$. Cell zone conditions entailed the impeller-fluid interface, which consisted of the impeller surface and the fluid regions around the impeller. Mesh interfaces and contact regions were confirmed to be the exact points where interactions occurred.

The movement of the impeller zone in the tankfluid region was modeled using a Multiple Reference Frame (MRF) approach that combines the computation of both stationary and moving frames. The two zones consists of well-defined boundaries. The moving zone comprised of the impeller and the shaft domains, rotating with an angular velocity of 600rpm along the y-axis. The tank-fluid zone together with the baffles and tank walls were set to the stationary frame (Pope, 2000).

The simulation was configured using Hybrid initialization technique before running the calculations with 200 iterations at a reporting interval of six and profile update of four cycles. The calculations were ascertained to have converged when the scaled residuals for each transport equation were below $10^{-4}$, within 1- 4 hours of computational. Velocity profiles 
were finally generated in CFD-post process to represent the effects of each impeller type. The simulations were executed using a $2.30 \mathrm{GHz}, 4 \mathrm{~GB}$ RAM, (Lenovo intel core i5) lap-top computer.

\section{CFD Post-Process Analysis}

\subsection{Flow Profiles in $r$ - $\theta$ Plane}

From the velocity streams generated (Figures 4 and 5), it can be seen that the fluid is well projected radially in both directions for both designs.

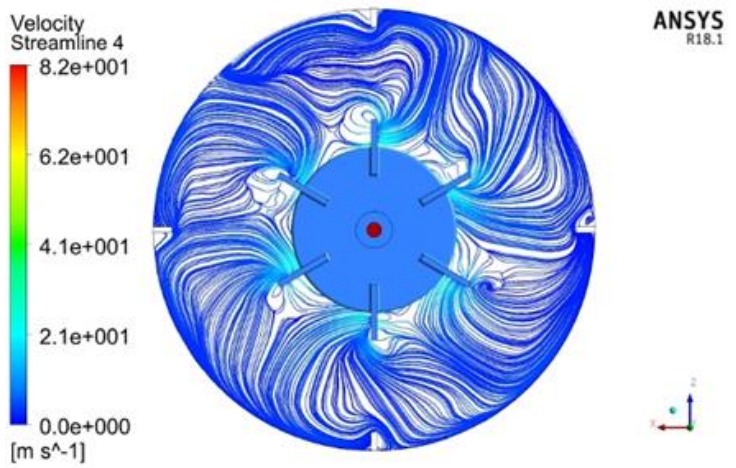

Fig. 4 Flow streamlines in $r-\theta$ plane of the Rushton turbine
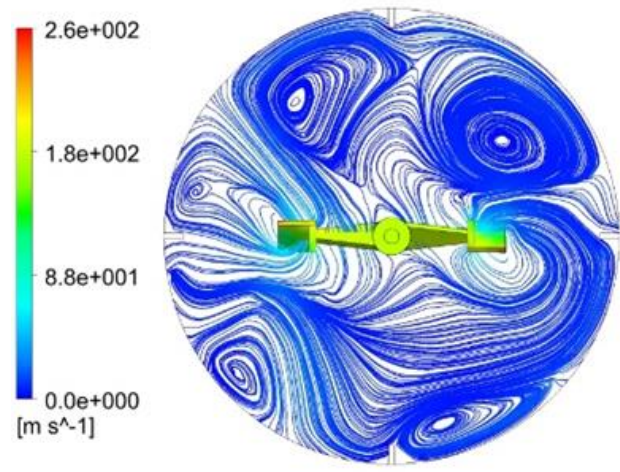

ANSYS

Fig. 5 Flow streamlines in $r-\theta$ plane of the counteraxial flow impeller

The flow patterns around the Rushton impeller region were generally well distributed due to its radial action on the fluid. This impeller was able to thrust the fluid from the center towards the walls of the tank and as the flow approached the wall regions, the velocity is observed to steadily drop. The counter-axial flow impeller is seen to cause recirculation loops indicating the fluid being projected to the walls at the inclined blade surface.

The effects of the baffles is clearly seen by the loops formed as the flow streams approach and leave them, culling in the region between baffles. The baffles intercepts the circular motion and cause turbulence in the fluid volume. This prevents vortex from forming around the shaft.
4.2 Flow Profiles in r-z Plane
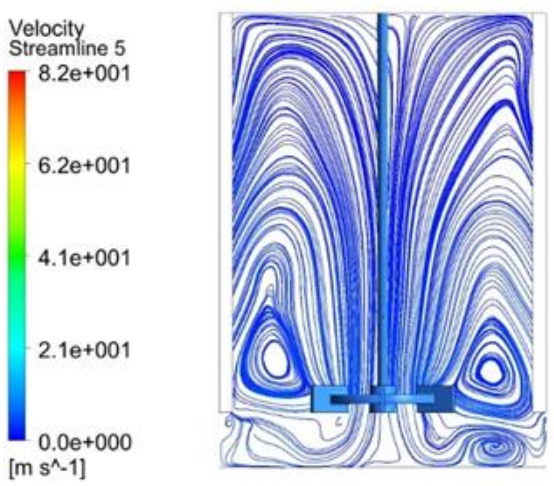

ANSYS

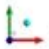

Fig. 6 Flow streamlines in r-z plane of the Rushton turbine
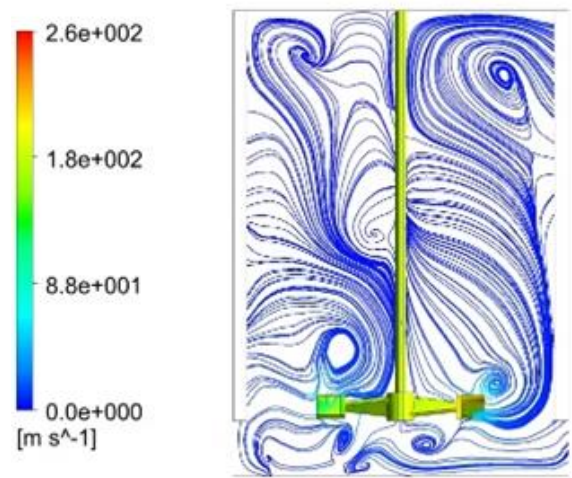

ANSYS

Fig. 7 Flow streamlines in r-z plane of the counter-axial flow impeller

The pumping action of the Rushton turbine is directed towards the wall of the tank. The flow streamlines results into axial movement when the radial flow meets the tank wall and then splits into upwards and downward streams. This creates two recirculation loops in both sides of the impeller. The recirculation loops are observed to form near the vessel walls and corners.

The counter axial flow impeller generates more recirculation loops both above and below the impeller, which improve mixing of substances. A circulation loop is formed in the lower middle part of the vessel by the counter-axial flow impeller due to the down pumping action of the inclined face. Above the impeller region, it can as well be observed that there is a turbulent streams in circular motion projected axial upwards. The fluid is discharged approximately at $45^{\circ}$ axially for the most part, both in the upward and downward directions. The mixing substances will thus, be better distributed at the central parts of the vessel as compared to the outer regions.

It was noted that the velocities caused by the counter-axial flow impeller in the upper regions of the tank and at the bottom of the tank appear greater than in the case of the Rushton turbine, indicating a rigorous mixing by this type of impeller. 


\subsection{Radial and Axial Velocity Characteristics}

These profiles are presented in the vertical and radial plane that contains the impeller. Figure 8 shows the axial profiles of the radial velocity component on the height of the tank. The discharge region, the lower section and the upper section can be defined from the shape. The profile of the Rushton turbine can be seen to be more outwardly projected due to the radial action of this impeller. The radial component of the counteraxial flow impeller is generally lean but a recirculation loop can be observed at the lower section. This would drive out mixing lodged at the bottom.

Figure 9 shows the axial profiles of the axial velocity component on the height of the tank. Two circulation regions can be seen on the upper and lower regions of the impeller.

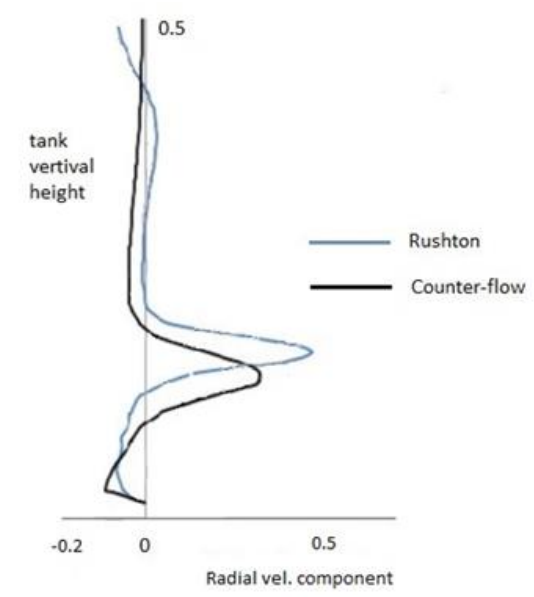

Fig. 8 radial velocity profile

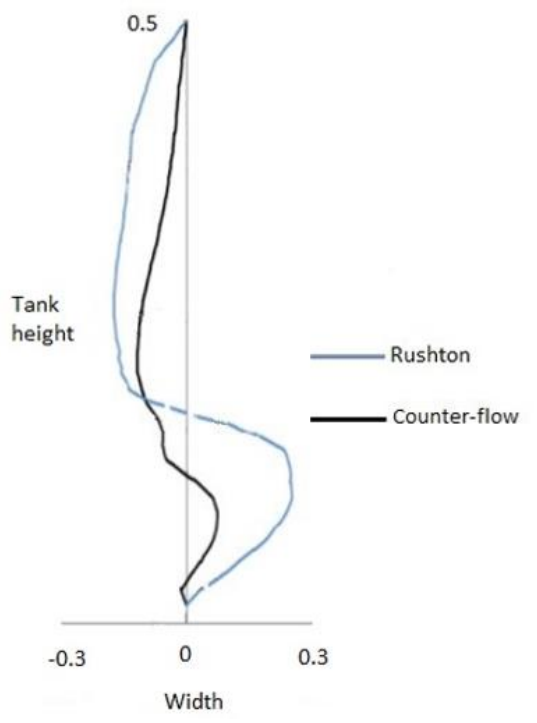

Fig. 9 axial velocity profile

The axial profile of the Rushton turbine is seen to extend more outwards from the shaft region. This impeller would act mostly on fluid further from the shaft. The counter-axial flow impeller is seen to direct the flow along the shaft region, due to the inclined planes facing both upwards and downwards.

\subsection{Validation of the Numerical Method.}

The profile obtained by the counter-axial flow impeller were validated using the experimental profile obtained by (Aubin, et al, 2001), who used a pitched blade turbine with $45^{\circ}$ incline, similar to one face of the counter axial impeller (Figure 10). The Comparison between the numerical results showed a good agreement, which proves the validity of the numerical method used in this work.

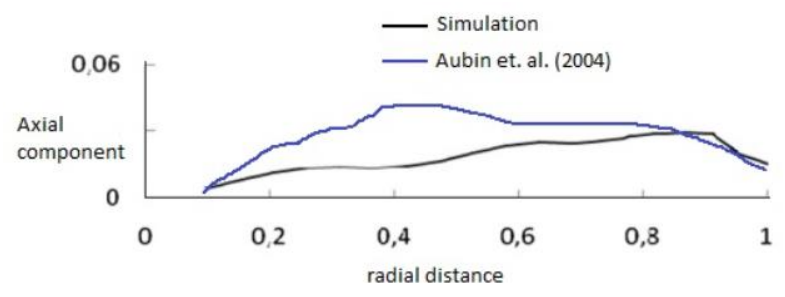

Fig. 10 Turbulent flow profile of counter axial impeller

The radial profile for the Rushton turbine were validated using the findings of (Ochieng and Maurice 2008), as shown in Figures 11 and 12.

The generated flow profiles in the radial direction were in good agreement as shown in figure below. The region close to the impeller shows a velocity component of between 0.4 and 0.5 . This indicates high turbulence intensity at the impeller region for both designs.

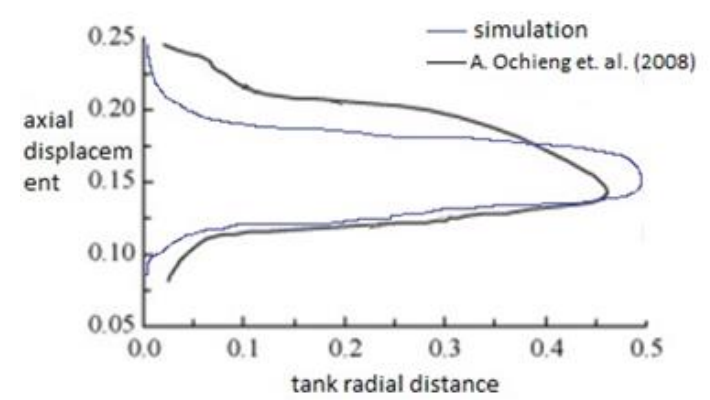

Fig. 11 Radial velocity flow field

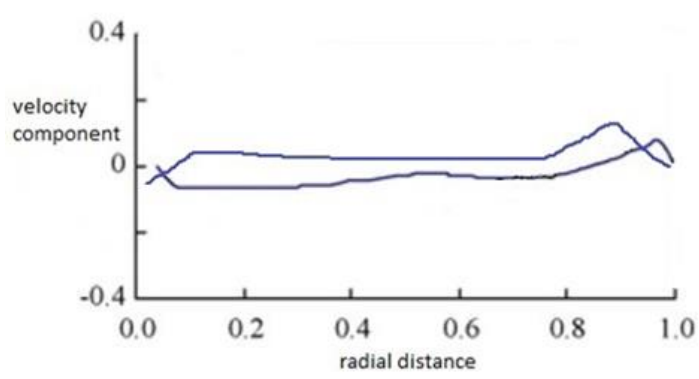

Fig. 12 axial velocity component 
The use of the turbulence models can provide a good basis for detailed understanding of generated flow in mixing vessels. Turbulent mixing in agitated vessels involve interactions between flows due to impeller blades, fluid and baffles. The circular motion of the fluid causes a complex recirculating flow in the tank, where the stationary baffles interact with the flow, improving the agitation. The shape of the recirculation loops depends of the impeller type.

From the obtained results, it was evident that the counter flow impellers exhibited better mixing results, which is essential in achieving homogeneity in agitation tanks. The new impeller design was, thus expected to increase mixing performance due to the counter flow characteristics. This outcome agrees well with the experimental findings as well as the explanation given by (McDonough, 1992) on the characteristics of the axial flow impellers.

\section{Conclusion}

In conclusion, we have studied the flow characteristics of a counter-axial flow impeller that would improve the turbulence characteristics in a stirred vessel, using CFD turbulent models. By simply changing the design of the conventional impeller types, better turbulence characteristics, which would improve the mixing process, can be achieved. The counter-axial flow impeller has been shown to circulate fluid more efficiently in both the up pumping and down pumping directions than the Rushton turbine. Achieving the best mixing designs will improve the quality of mixing and establish a good degree of homogeneity, as a function of the design. In the future, we propose the use of more advanced turbulence models such as the LES model for improved accuracy. It would also be worthwhile performing experiments with a multi stage combination of this impeller for improved mixing.

\section{References}

F. Delvigne, J. Destain, P. Thonart, (2005), Structured Mixing Model for Stirred Bioreactors: An Extension to the Stochastic Approach, Chemical Engineering Journal, 113, 112.

H. K. Versteeg, W. Malalasekera, (2007), An Introduction to Computational Fluid Dynamics. In The Finite Vol. Method, 2nd ed. Pearson Education: New York.

M. Sommerfeld, S. Decker, (2003), Proceedings of the 11th European Conference on Mixing, Bamberg, p. 1.
G. Chun-Yan, W. Jia-Jun, G. Xue-Ping, F. Lian-Fan, (2014), CFD simulation and PIV measurement of the flow field generated by modified pitched blade turbine impellers, Chemical engineering research and design, 92, 1027-1036.

C. Kuncewicz, M. Pietrzykowski, (2006), Hydrodynamic model of a mixing vessel with pitched-blade turbines. Chem Eng Sci, 56, 4659-72.

T. Kumaresan, J. B. Joshi, (2006), Effect of impeller design on the flow pattern and mixing in stirred tanks, Chem Eng Sci, $115,173-93$.

M. H. Vakili, N. M. Esfaharry, (2009), CFD analysis of turbulence in a baffled stirred tank, a three-compartment model, Chem Eng Sci, 64, 351-62.

J. Aubin, C. Xuereb, (2006), Design of multiple impeller stirred tanks for the mixing of highly viscous fluids using CFD, Chemical Engineering Science, 61, (9), 2913-2920.

M. Jahoda, K. M. Moste, A. Kukukova, (2007), CFD modelling of liquid homogenization in stirred tanks with one and two impellers using large eddy simulation. Chemical Engineering Research and Design, 85, (5), 616-625.

M. Musgrove, S. Ruszkowski, H. Van den Akker, J. Derksen, (2000), Influence of impeller type and agitation conditions on the drop size of immiscible liquid dispersions. Proceedings of the 10th European Conference on Mixing; Elsevier Science, Amsterdam,165-172.

A.W. Nienow, (1996), Mixing studies: a comparison of Rushton turbine with some modern impellers, Chem. Eng. Res. Des., 74, 417-423.

P. Bonvillani, M. P. Ferrari, E. M. Ducrós, J. A. Orejas, (2006), Theorical and experimental study of the effects of scale-up on mixing time for a stirred-tank bioreactor, Brazilian Journal of Chemical Engineering, 23, 01.1.

M.A. Leschziner, D. Drikakis, (2002), Turbulence and turbulent flow computation in aeronautics. The Aeronaut J., 106, (1061), 349-84.

ANSYS Fluent User Guide. Available online: http://www.ansys.fem.ir/ansys_fluent (accessed on 17th December 2017).

E. L. Paul, V. A. Atiemo-Obeng, S. M. Kresta, (2004), Handbook of Industrial Mixing: Science and Practice, WileyInterscience: New York.

C.P.K. Dagadu, Z. Stegowsk, L. Furman, E.H.K. Akaho, K.A. Danso, (2014), Determination of Flow Structure in a Gold Leaching Tank by CFD Simulation, Journal of Applied Mathematics and Physics, 2, 510-519.

L. M. Oshinowo, A. Bakker, (2002), CFD Modeling of Solids Suspensions in Stirred Tanks. Presented at the TMS Annual Meeting, Seattle, WA, 215.

S. Pope, (2000), Turbulent flows. Cambridge University Press.

J. Aubin, P. Mavros, D. F. Fletcher, J. Bertrand, C. Xuereb, (2001), Effect of axial agitator configuration (up-pumping, down-pumping, reverse rotation) on flow patterns generated in stirred vessels. Trans IChemE, 79, 845-56.

A. Ochieng, S. O. Maurice (2008), Homogenization energy in a stirred tank, Chemical Engineering and Processing, 47, 1853-1860.

R. J. McDonough, (1992), Mixing for the Process Industries, Van Nostrand Reinhold: New York. 